\section{Posición del agujero dentario inferior en la rama ascendente en huesos mandibulares secos de adultos.}

Gutierrez-Ventura $F^{\prime}$, Tataje-Vivanco $Y^{2}$. Posición del agujero dentario inferior en la rama ascendente en huesos mandibulares secos de adultos. Rev Estomatol Herediana. 2012; 22(3): 152-7.

\section{RESUMEN}

Objetivos: El presente estudio consistió en observar y definir la ubicación del agujero dentario inferior en la rama ascendente de 53 mandíbulas secas de adultos. Material y métodos: La muestra fue recolectada del laboratorio de anatomía de la Facultad de Estomatología de la UPCH. Se evaluó y midió las distancias del agujero dentaria inferior hacia los bordes limitantes de la rama mandibular, es decir la distancia anterior, posterior, superior e inferior y establecer relación entre las medidas obtenidas de la rama derecha e izquierda. Resultados: Los resultados fueron para la distancia anterior $1.72 \mathrm{~cm}$ en la rama derecha y $1.68 \mathrm{~cm}$ en la rama izquierda; para la distancia posterior 1.40 $\mathrm{cm}$ en la rama derecha y 1.39 en la rama izquierda; para la distancia superior $1.77 \mathrm{~cm}$ en la rama derecha y $1.74 \mathrm{~cm}$ en la rama izquierda; para la distancia inferior $2.48 \mathrm{~cm}$ en la rama derecha y 2.45 $\mathrm{cm}$ en la rama izquierda. Conclusiones: Se hallaron diferencias estadísticamente significativas para las distancias anterior, superior e inferior.

Palabras clave: MANDÍBULA, HUESOS FACIALES, INERVACIÓN, MAXILAR.

Mandibular position hole ramus in mandibular bone dry in adults.

\section{ABSTRACT}

Objectives: This study was to observe and define the location of the mandibular hole in the ascending limb of 53 adult dry mandibles. Material and methods: The sample was collected from the anatomy laboratory of the Faculty of Dentistry of the UPCH. We evaluated and measured the distances from the hole to edge lower dental ramus constraints, ie the distance front, back, top and bottom and establish relationship between the measurements obtained from the right and left branch.

Results: The results were for the previous distance in the right branch $1.72 \mathrm{~cm}$ and $1.68 \mathrm{~cm}$ in the left branch, for the further distance $1.40 \mathrm{~cm}$ in the right arm and 1.39 in the left branch, for superior distance $1.77 \mathrm{~cm}$ in the right branch and $1.74 \mathrm{~cm}$ in the left branch, for the shortest distance in the right branch $2.48 \mathrm{~cm}$ and $2.45 \mathrm{~cm}$ in the left branch.

Conclusions: Statistically significant differences were found for distances above, top and bottom.

\section{Fredy Gutierrez Ventura ${ }^{1}$ Yarim Tataje Vivanco ${ }^{2}$}

${ }^{1}$ Magister en Estomatología.

Cirujano Oral y Maxilofacial.

Ortodoncista.

Docente Asociado. Facultad de Estomatología Roberto Beltrán. Universidad Peruana Cayetano Heredia.

Lima Perú

2 Cirujano Dentista Práctica Privada

\section{Correspondencia:}

Fredy Gutierrez Ventura

Calle da Vinci 528 Urb. La calera de

la Merced.

Surquillo Lima

Teléfono: 2715506 - 998274209

Email: fredy.gutierrez@upch.pe

Key words: MANDIBLE, FACIAL BONES, INNERVATION, MAXILLA.

\section{Introducción}

La importancia de conocer la anatomía del hueso mandibular es el comienzo y la base del saber del sistema estomatognático, pues guían los procedimientos realizados por el odontólogo desde una simple técnica de anestesia hasta procedimientos quirúrgicos complejos.

Sabemos que la mandíbula es el único hueso móvil facial, constituye por si solo todo el macizo óseo inferior de la cara, esta comprende un cuerpo impar y dos ramas ascendentes, estas últimas están marcadas fundamentalmente por la presencia del agujero y conducto del nervio dentario inferior. Dicho agujero adquiere evidente jerarquía en cirugía oral, ya que es el punto crítico para la anestesia regional del nervio dentario inferior. Según los anatomistas el orificio dentario inferior esta situado a igual distancia de los cuatro bordes de la rama y en la prolongación de un plano imaginario que pasa por la superficie triturante de las terceras molares.

La mandíbula constituida por un tejido esponjoso situada entre dos láminas de tejido compacto que en su interior presenta el conducto mandibular comienza en la cara medial de la rama a nivel del foramen mandibular y termina a nivel del orificio mentoniano $(1,2)$.

Tal como le explica Latarjet, el agujero dentario inferior es una estructura muy importante, presenta en su borde anterior e inferior un borde agudo que luego pasara a formar una lengüeta ósea conocida como la espina Spix (3).

López Aranz JS observó una saliente ósea llamada espina spix en el agujero dentario inferior se donde se inserta el ligamento esfenomandibular (4). El agujero dentario se encuentra localizado a las siguientes distancias de la rama mandibular: 
A $18 \mathrm{~mm}$ del borde anterior, a 15 $\mathrm{mm}$ del borde anterior, a $12 \mathrm{~mm}$ de la escotadura sigmoidea y a $8 \mathrm{~mm}$ de la línea oblicua interna.

Mbajiorgu realizó estudios sobre la posición del foramen en 38 mandíbulas de adultos negros, se midió el agujero teniendo como resultado que el diámetro en cuanto al ancho es de $4,64 \mathrm{~mm}$ y de $6 \mathrm{~mm}$ de altura. También se obtiene resultados en relación con la posición del foramen mandibular con respecto al plano oclusal, concluyendo que $47 \%$ se encuentra al mismo nivel; 29,4 $\%$ por encima del plano oclusal y $23,5 \%$ por debajo del plano (5).

Demostraron que la distancia del agujero dentario a el borde anterior es de 18,95+/- 0,41, distancia del agujero dentario hacia el borde posterior es de 14,30+/- 0,35, distancia del agujero dentario hacia el borde superior es de 22,50 +/- 0,50 y distancia del agujero dentario hacia el borde inferior es de 28,44 +/- 0,65.

Se realizaron medidas transversales y longitudinales en la rama mandibular donde se obtuvo los siguientes resultados: Ancho de la rama mandibular Derecha: $3,1 \mathrm{~cm}$ (D.S. $=0,32)$ e Izquierda: $2,97 \mathrm{~cm}$ (D.S. $=0,31)$. Altura de la rama mandibular: Derecha: $1,63 \mathrm{~cm}$ (D.S. $=0,25)$ e Izquierda: $1,59 \mathrm{~cm}$ (D.S. $=0,31)$. Borde anterior de la rama mandibular al borde anterior del foramen mandibular: derecha: $1,70 \mathrm{~cm}$. (D.S. $=0,20)$ e izquierda: $1,69 \mathrm{~cm}(\mathrm{D} . \mathrm{S} .=0,20)$. Borde basal de la mandíbula al punto más inferior del foramen mandibular: derecha: 2,46 cm. (D.S. $=0,29)$ e Izquierda: $2,44 \mathrm{~cm}$. (D.S. $=0,30$ )

Kaffe y colaboradores, realizaron un estudio en 100 huesos mandibulares de adultos con dentición permanente y en sus respectivas radiografías haciendo uso de
2 máquinas panorámicas (6). Con respecto a las mediciones en los huesos mandibulares tuvo como resultado las siguientes medidas: ancho de la rama mandibular en su porción más estrecha: $31,22 \mathrm{~mm}$, distancia desde el punto más superior del agujero dentario inferior hacia el punto más inferior de la escotadura sigmoidea: $17,92 \mathrm{~mm}$, distancia del borde anterior mandibular (línea oblicua externa) hasta el borde más inferior del agujero dentario inferior: 17,28 mm.

Fontoura y colaboradores, realizaron mediciones de la posición del agujero dentario inferior visualizando y estimando sus distancias con respectos a los bordes mandibulares de 140 huesos mandibulares, con el propósito de proporcionar parámetros anatómicos y radiográficos que permitan la realización de la osteotomía vertical de rama sin dañar del agujero dentario inferior (7).

Así tenemos la distancia del borde posterior del agujero dentario inferior al borde más posterior de la rama: $10,04 \mathrm{~mm}$, distancia del borde superior del agujero dentario inferior a el punto mas inferior de la escotadura sigmoidea: $16,8 \mathrm{~mm}$, ancho total mandibular (desde el borde anterior a el borde posterior de la rama posterior): $31,03 \mathrm{~mm}$ y altura total de la rama mandibular (desde el punto más inferior de la escotadura sigmoidea hasta el borde basal mandibular): 49,4 $\mathrm{mm}$.

La investigación tiene como propósito establecer parámetros longitudinales-anatómicos con respecto a la posición del agujero dentario inferior en la rama ascendente que permitan realizar procedimientos anestésicos y quirúrgicos con mayor exactitud para no dañar el nervio dentario inferior.

\section{Material y métodos}

El diseño de este estudio es observacional y transversal.

La muestra estuvo conformada por 90 mandíbulas escogidas del laboratorio de anatomía de la Facultad de Estomatología Roberto Beltrán de la Universidad Peruana Cayetano Heredia, que pasaron por una revisión estructural y morfológica. La muestra se determinó por una prueba piloto que se realizó mediante la siguiente fórmula para obtener el número de mandíbulas a utilizar:

$\mathrm{N}=\left(\begin{array}{lll}\mathrm{Z}^{2} \mathrm{X} & \mathrm{Sx}^{2}\end{array}\right) / \mathrm{E} 2$

Nivel de significancia:

$\mathrm{Z}=1.96$ si $\mathrm{Sx}=0,05$

Error muestral: $\mathrm{S}=0,1$

Desviación estándar obtenido:

$\mathrm{Sx}=0,21$

El tamaño mínimo de la muestra fue de 18 mandíbulas. Los criterios de Inclusión fueron:

- Huesos mandibulares de adultos con presencia de piezas posteriores (molares).

- Mandíbulas que presenten las 2 ramas ascendentes.

- Integridad de las ramas ascendentes mandibulares.

- Buen estado de los bordes anterior, posterior, superior e inferior de ambas ramas mandibulares.

- Conservación integra del agujero dentario inferior.

- Escotadura sigmoidea en buen estado de conservación. Es indistinta del género y edad de los huesos mandibulares.

De acuerdo a los criterios de inclusión se obtuvo la conformidad solo en 53 mandíbulas, para las mediciones se tomó en cuenta la técnica de medición que utilizó en la publicación de Kaffe y col (6): a. Distancia del borde anterior del foramen mandibular a borde anterior de la rama ascendente, $b$. Distancia del borde posterior del foramen 
mandibular a borde posterior de la rama ascendente c. Distancia del borde superior del foramen mandibular al borde superior de la rama ascendente. D. Distancia del borde inferior del foramen mandibular al borde basal mandibular.

\section{Procedimientos y Técnicas:}

Recolectada la muestra se procedió a marcar los puntos de referencia en ambas ramas ascendentes de cada mandíbula:

Punto A: Punto más superior del agujero dentario inferior

Punto B: Punto más anterior del agujero dentario inferior

Punto C: Punto más posterior del agujero dentario inferior

Punto D: Punto más inferior del agujero dentario inferior

Punto E: Punto más profundo de la escotadura sigmoidea

Punto F: Punto localizado en el borde anterior en la región más cóncava de la rama mandibular.

Punto G: Punto localizado en el borde posterior en la región más cóncava de la rama mandibular.

Punto H: Punto localizado en el borde inferior de la rama mandibular.

Luego de marcar los puntos referenciales se procedió a realizar los trazos que serán marcados sobre cada rama ascendente de los huesos mandibulares

A-E.- Trazo vertical que describe la distancia entre el borde superior del agujero dentario hacia el borde superior de la rama representada por el punto mas profundo de la escotadura sigmoidea. B-F.- Trazo horizontal: es la distancia entre el borde más anterior del agujero dentario inferior hacia el borde anterior de la rama mandibular.

C-G.- Trazo horizontal desde la distancia entre el borde más posterior del agujero dentario inferior hacia el borde posterior de la rama mandibular.

D-H.- Trazo vertical desde la distancia entre el borde inferior del agujero dentario inferior hacia el borde inferior de la rama representada por un trazo que pasa por la mayoría de puntos del borde basal.

Las mediciones se realizaron con una regla milimetrada flexible donde la unidad mínima de medida es la mitad de milímetro.

El orden del proceso de medición se realizó de la siguiente manera:

Trazo A-E, Trazo B-F, Trazo C-G, Trazo D-H. Luego de tener todas las mediciones se procedió a realizar una segunda medición con la finalidad de confirmar la primera. La segunda medición se realizó desconociendo las medidas ya tomadas anteriormente.

Finalmente teniendo tanto las primeras como las segundas mediciones, se procedió a comparar ambas mediciones.

Cuando se presentaron alguna diferencia entre las medidas se promedió ambas mediciones y esa fue tomada como la definitiva.

\section{Plan de análisis}

Obtención de promedios y desviaciones estándar de las variables, comparación de las variables de ambos ramas derecha e izquierda a través de la prueba $T$ de studens para muestras pareadas y presentación de los resultados en tablas y gráficas respectivas.

\section{Resultados}

Se realizó mediciones longitudinales en 55 huesos secos mandibulares de adultos.

La distancia promedio entre el borde anterior del agujero dentario inferior al borde anterior de la rama ascendente del lado derecho se encontró que fue de $1,72 \mathrm{~cm}$. (D.S. $=0,18)$ y el promedio del lado izquierdo fue de $1,68 \mathrm{~cm}$.
(D.S.=0,18) (Gráfico 1).

La distancia promedio entre el borde posterior del agujero dentario inferior al borde posterior de la rama ascendente del lado derecho se encontró que fue de $1.40 \mathrm{~cm}(D . S .=0,19)$ y el promedio del lado izquierdo fue de $1,39 \mathrm{~cm}$ (D.S. $=0,19)$ (Gráfico 2).

La distancia promedio entre el borde superior del agujero dentario inferior al borde superior de la rama ascendente del lado derecho se encontró que fue de $1,77 \mathrm{~cm}$. (D. $S=0,25)$ y el promedio del lado izquierdo fue de1,74 cm (D.S.= 0,29) (Gráfico 3).

La distancia promedio entre el borde inferior del agujero dentario inferior al borde inferior de la rama ascendente del lado derecho se encontró que fue de $2,48 \mathrm{~cm}$. (D.S. $=0,32)$ y el promedio del lado izquierdo fue de $2,45 \mathrm{~cm}$. (D.S.=0,33) (Gráfico 4).

Según la prueba $\mathrm{T}$ para muestras pareadas, se halló diferencias estadísticamente significativas para las distancias AEd - AEi (borde superior del agujero dentario inferior al borde superior de la rama ascendente), BFd - Bfi (borde anterior del agujero dentario inferior al borde anterior de la rama ascendente) DHd -DHi (borde inferior del agujero dentario inferior al borde inferior de la rama ascendente representada por la basal mandibular).

\section{Discusión}

En el estudio se determinó la posición del agujero dentario inferior en huesos secos mandibulares de adultos, La distancia promedio del agujero dentario inferior hacia el borde anterior de la rama ascendente derecha se obtuvo $1,72 \mathrm{~cm}$. (D.S= $0,18)$ y para el lado izquierdo 1,68 $\mathrm{cm}$. (D.S $=0,18)$ coincidiendo con el estudio realizado por Langston 


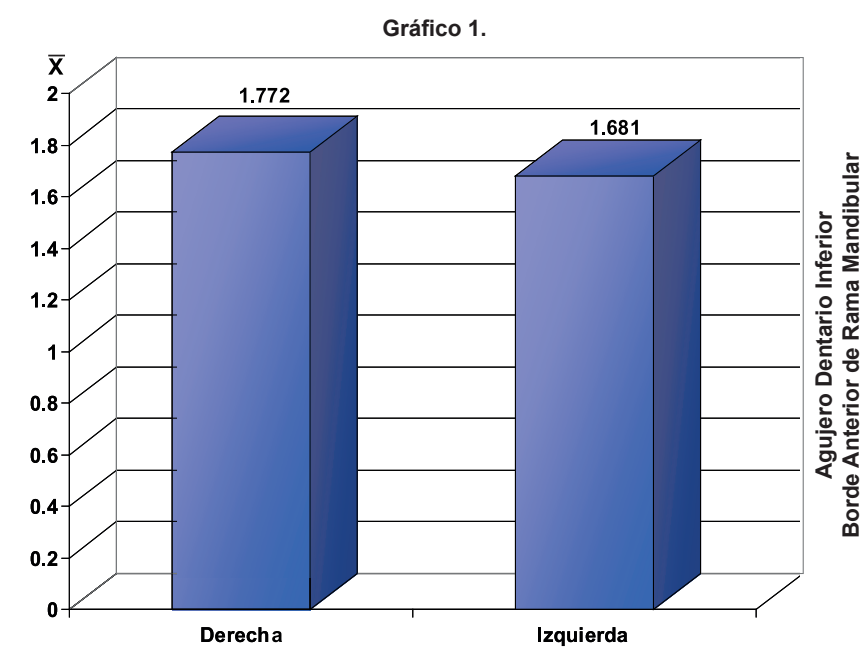

Distancia Anterior: Agujero Dentario Inferior - Borde Anterior de Rama

Gráfico 2

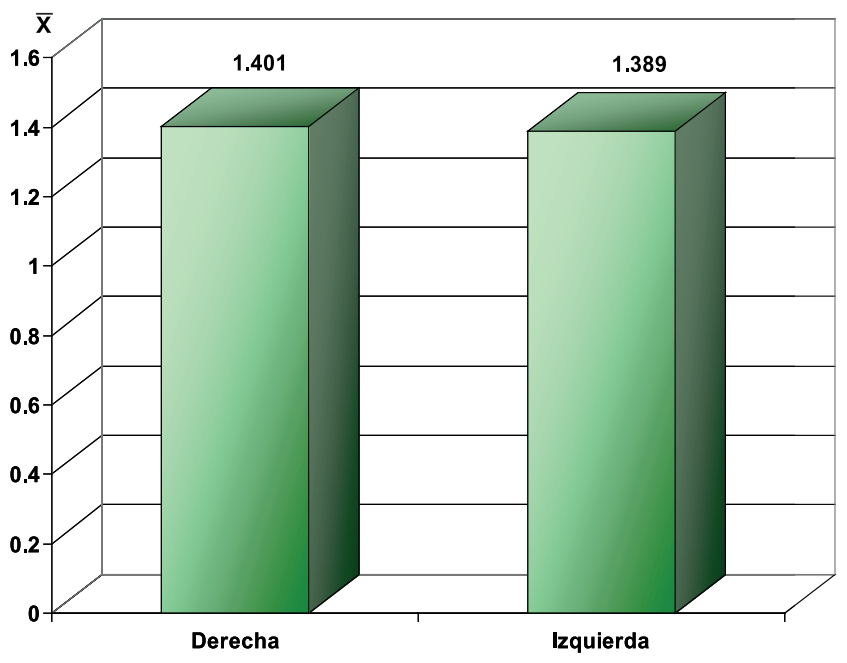

Distancia Posterior: Agujero Dentario Inferior - Borde Posterior Rama

Gráfico 3

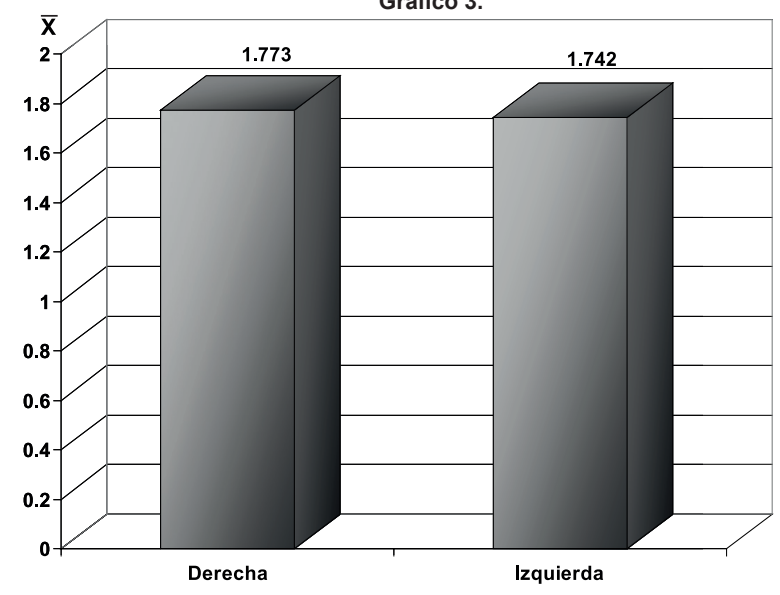

Distancia Superior Agujero Dentario Inferior-Escotadura Sigmoidea

100 huesos mandibulares de adulto, obtuvo como resultado en la distancia anterior de la rama ascendente la medida de $1,72 \mathrm{~cm}$., en dicho estudio no presentaron los resultados por cada rama ascendente de una misma mandíbula (6).

Mientras que los resultados obtenidos en relación a la distancia anterior, no coincide con los encontrados por Mbajiorgu en su estudio sobre la posición del foramen mandibular en 38 huesos mandibulares secos de adultos de raza negra (5), donde demuestran que la distancia anterior es de $1,89 \mathrm{~cm}$. +/-0,05. Así mismo, no concuerda con los resultados presentados por Afsar y colaboradores, en su investigación realizada a 70 huesos mandibulares donde se halló que el promedio de esta distancia fue de 2,02 cm (8).

Por otro lado, los resultados con respecto a la medida posterior no concuerdan con Afsar y colaboradores en su estudio realizado a 70 mandíbulas el cual obtuvo que el promedio posterior fue de $1,3 \mathrm{~cm}$ (8). Del mismo modo, se observa discrepancia con los resultados hallados por Fontoura y colaboradores en este estudio se realizaron mediciones en cuanto a la posición del agujero dentario inferior estimando sus distancias con respecto a los bordes de la rama en 140 huesos mandibulares secos obteniendo así la distancia del borde posterior de $1,0 \mathrm{~cm} .(7)$; esta discrepancia se puede deber por que en dicho estudio, la distancia posterior estaba establecida desde el borde mas posterior del foramen trazando una línea recta horizontal hacia el borde posterior de la rama ascendente; mientras que en el presente estudio la distancia posterior se trazó desde el borde mas posterior del foramen trazando una línea hacia el punto mas cóncavo del borde posterior

os mandibulares, se tuvo como resultado $1,70 \mathrm{~cm}$. (D.S=0,20) para el lado derecho y para el lado izquierdo 1,69 cm. (D.S=0,20). (9),

A su vez Kafé y colaboradores, en su investigación realizada en 


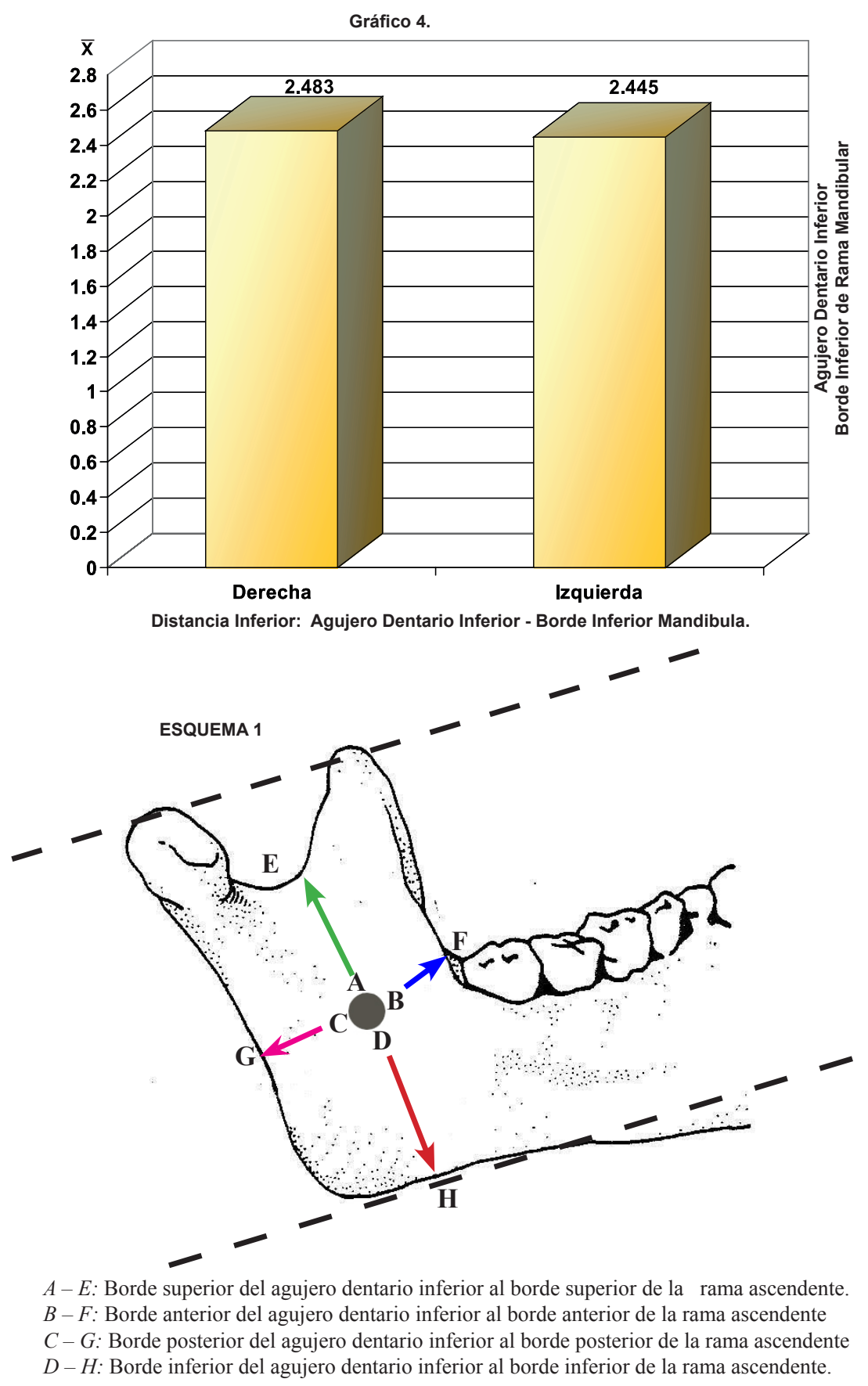

de la rama ascendente representada por la basal mandibular).

Con respecto a la distancia promedio del agujero dentario inferior hacia el borde superior de la rama ascendente derecha se obtuvo 1,77 cm. (D. $S=0,25)$ y para el lado izquierdo $1,74 \mathrm{~cm}$. (D. $S=0,29$ ) estos resultados son similares a los presentados por Kaffe y colaboradores, que realizaron un estudio en cm (5).Afsar y colaboradores en su publicación dan como resultado para esta medida $1,99 \mathrm{~cm}(8)$.

\section{Conclusiones}

En relación a los objetivos planteados, se establece las siguientes conclusiones:

La distancia del borde anterior del agujero dentario inferior con respecto al borde anterior de la rama ascendente mandibular derecha fue de $1,72 \mathrm{~cm}$. (D.S $=0,18$ )

La distancia del borde anterior del agujero dentario inferior con respecto al borde anterior de la rama ascendente mandibular izquierdo fue de $1,68 \mathrm{~cm}$. (D.S=0,18)

La distancia del borde posterior del agujero dentario inferior con respecto al borde posterior de la rama ascendente mandibular derecho fue de $1,40 \mathrm{~cm}$. (D.S=0,19)

La distancia del borde posterior del agujero dentario inferior con respecto al borde posterior de la rama ascendente mandibular izquierdo fue de $1,39 \mathrm{~cm}$. (D.S=0,19)

La distancia del borde superior del agujero dentario inferior con respecto al borde superior de la rama ascendente mandibular derecho fue de $1,77 \mathrm{~cm}$. (D.S=0,25)

La distancia del borde superior del agujero dentario inferior con respecto al borde superior de la rama ascendente mandibular izquierdo fue de $1,74 \mathrm{~cm}$. (D.S=0,29)

La distancia del borde inferior del agujero dentario inferior con respecto al borde inferior de la rama ascendente mandibular derecha fue de $2,48 \mathrm{~cm}$. (D.S $=0,32$ )

La distancia del borde inferior del agujero dentario inferior con respecto al borde inferior de la rama ascendente mandibular izquierda fue de $2,45 \mathrm{~cm}$. (D.S=0,33)

Existe una diferencia estadísticamente significativa con respecto a la 
distancia anterior, superior e inferior del agujero dentario inferior a sus respectivos bordes mandibulares.

No hay una relación estadísticamente significativa con respecto a la distancia posterior de ambas ramas ascendentes mandibulares.

\section{Referencias bibliográficas}

1. Figun M. Anatomía Odontológica funcional y aplicada. Editorial El ateneo 2 edición Buenos Aires: Editorial El ateneo; 2001 p. 23-7; 273-7.

2. Nakajima K, Onoda Y, Okada M, Abe S, Ide Y. A study of the internal structure of the mandibular ramus in Japanese. The Bull Tokyo Dent Colleg; 1998, 39(1):57-65.

3. Latarjet L. Anatomía Humana. Editorial médica panamericana 2 edición, 1989; p 97-9.
4. Lopez Aranz JS. Cirugía Oral. Ed Interamericana Mc Graw Hill. Edit. Panamericana. $1^{\circ}$ edición, 1988; p 36-9.

5. Mbajiogu EF. A study of the position of the mandibular foramen in adult black Zimbabwean mandibles. Cent Afr J Med. 2000;46(7):184-90.

6. Kaffe I, Ardekian L, Gelenter I. Location of the mandibular foramen in panoramic radiographs. Oral Surg Oral Med Oral Pathol. 1994;78(5):6629.

7. Fontoura RA, Vasconcellos HA, Siquera AE. Morphologic basis for the intraoral vertical ramus osteotomy, anatomic and radiographic localization of the mandibular foramen. J Oral Maxillofac Surg. 2002;60(6):660-5.
8. Afsar A, Haas, Rossouw. Radiographic localization of mandibular anesthesia landmarks. Oral Surg Oral Med Oral Pathol. 1998; 86(4): 234-41.

9. Langston JR, Tebo HG. The incidence and relationship of the lateral ramus prominence to the mandibular foramen. Oral Surg Oral Med Oral Pathol; 1977; 44 (2): 190-6. 